\title{
Efficacy of coblation versus radiofrequency thermocoagulation for the clinical treatment of trigeminal neuralgia
}

\author{
Yanda Lin $^{1}$, Jiaxiang $\mathrm{Ni}^{1}$, Xinlu Zuo ${ }^{2}$, Liqiang Yang ${ }^{1}$, Liangliang $\mathrm{He}^{1}$, Yuanzhang Tang ${ }^{1}$, Chengli Sun ${ }^{1}$ \\ ${ }^{1}$ Department of Pain Management, Xuanwu Hospital, Capital Medical University, Beijing, China \\ ${ }^{2}$ Department of Nursing, Chengde Nursing Vocational College, Chengde, China
}

Videosurgery Miniinv 2020; 15 (4): 620-624

DOI: https://doi.org/10.5114/wiitm.2020.92409

\begin{abstract}
Introduction: Postoperative facial numbness is the main complication of radiofrequency thermocoagulation in treating trigeminal neuralgia, which could seriously affect the surgical efficacy. This problem is expected to be resolved by coblation technique.

Aim: To compare the long-term efficacy and safety of coblation and percutaneous trigeminal radiofrequency thermocoagulation (PT-RFT) under fluoroscopic guidance in the treatment of trigeminal neuralgia (TN).

Material and methods: A case-control prospective study was carried out. Patients with TN were randomly scheduled to receive coblation or PT-RFT. Both surgical procedures were performed under fluoroscopic guidance. The data, including the degree of pain, pain relief and complications, were recorded during follow-up evaluation, which was performed on the first day and at the end of the first month, third month, sixth month and first year after surgery. Results: A total of 50 patients were enrolled in this study, with 25 patients in each group. The visual analog scale (VAS) scores in both groups at each time point after surgery were significantly lower compared with before surgery $(p<0.05)$. There were no significant differences in VAS scores or pain relief between the two groups at any time point after surgery $(p>0.05)$. However, patients in the PT-RFT group exhibited greater facial numbness after surgery $(p<0.05)$. For other complications, there were no significant differences between the two groups $(p>0.05)$.

Conclusions: Coblation and PT-RFT showed similar effectiveness in reducing pain; however, coblation was associated with a lower rate of postoperative facial numbness. Therefore, coblation may be a better treatment option for $T N$.
\end{abstract}

Key words: trigeminal neuralgia, percutaneous trigeminal radiofrequency thermocoagulation, coblation, facial numbness.

\section{Introduction}

Idiopathic trigeminal neuralgia (ITN) is severe chronic neuropathic facial pain, characterized by recurrent episodes of intense, lancinating pain localized to dermatomes of the face innervated by the trigeminal nerve [1]. Oral medication, rapid and convenient, is the first-line therapy for ITN [2]. However, for medication-resistant or medication-intolerant ITN, surgical treatment needs to be considered [3].
Because of minimal trauma and long-term pain relief, percutaneous trigeminal radiofrequency thermocoagulation (PT-RFT) has become a primary minimally invasive surgical treatment modality [4]. However, the technique is associated with a high incidence of post-operative facial numbness because of nerve injury caused by the high temperatures needed for thermocoagulation [5]. In comparison, coblation has been reported to produce only slight numbness when used to ablate the thoracic paraver-

\section{Address for correspondence}

Jiaxiang Ni PhD, Dr. Liangliang He, Department of Pain Medicine, The Capital Medical University Xuan Wu Hospital,

e-mail: nijiaxiang@hotmail.com, Ilhe1981@126.com 
tebral nerve [6]. The relatively low working temperature $\left(40-70^{\circ} \mathrm{C}\right)$ and the short thermal penetration distance $(\sim 1.0 \mathrm{~mm})$ of coblation might help preserve neural function.

\section{Aim}

In our previous study, we provided preliminarily data showing that coblation can reduce the degree of postoperative facial numbness in patients with ITN in the short term [7]. In the present study, we further evaluate the long-term efficacy and safety of ablating the trigeminal ganglion with coblation technology under fluoroscopic guidance.

\section{Material and methods}

The randomized, prospective, clinical study was approved by the institutional Ethics Examining Committee of Human Research. A total of 56 patients with TN provided written informed consent, and were randomly scheduled to receive coblation (coblation group) or PT-RFT (RFT group) of the trigeminal ganglion between January, 2017 and June, 2018. ITN was di- agnosed according to the criteria of the International Classification of Headache Disorders-II (2004).

After entering the sterile operating room, all patients received standard monitoring, that is, heart and respiratory rate, and fingertip digital oximetry. A $0.5 \mathrm{~g}$ dose of atropine was administered intravenously $5 \mathrm{~min}$ prior to surgery to block the trigeminal-baroreceptor reflex, and a $50 \mu \mathrm{g}$ dose of fentanyl was injected intravenously to provide analgesia. No other sedatives were given, to maintain consciousness and responsiveness to nerve root stimulation during surgical procedures.

All surgical procedures were performed under fluoroscopic guidance, with the patient in the supine position. A soft pad of $10 \mathrm{~cm}$ thickness was placed under the neck to retroflex the head approximately $45^{\circ}$. Fluoroscopic guidance was performed with the OEC 9900 Elite C-Arms (the American, GE). The preset coronal and sagittal angles of the $\mathrm{X}$-ray tube to reveal the foramen ovale (FO) were $10^{\circ}$ and $10^{\circ}$, pre-operatively (Photo $1 \mathrm{~B}$ ).

After clearly visualizing the FO, an 18-gauge, 8-cm, guide needle was inserted through the sterilized skin
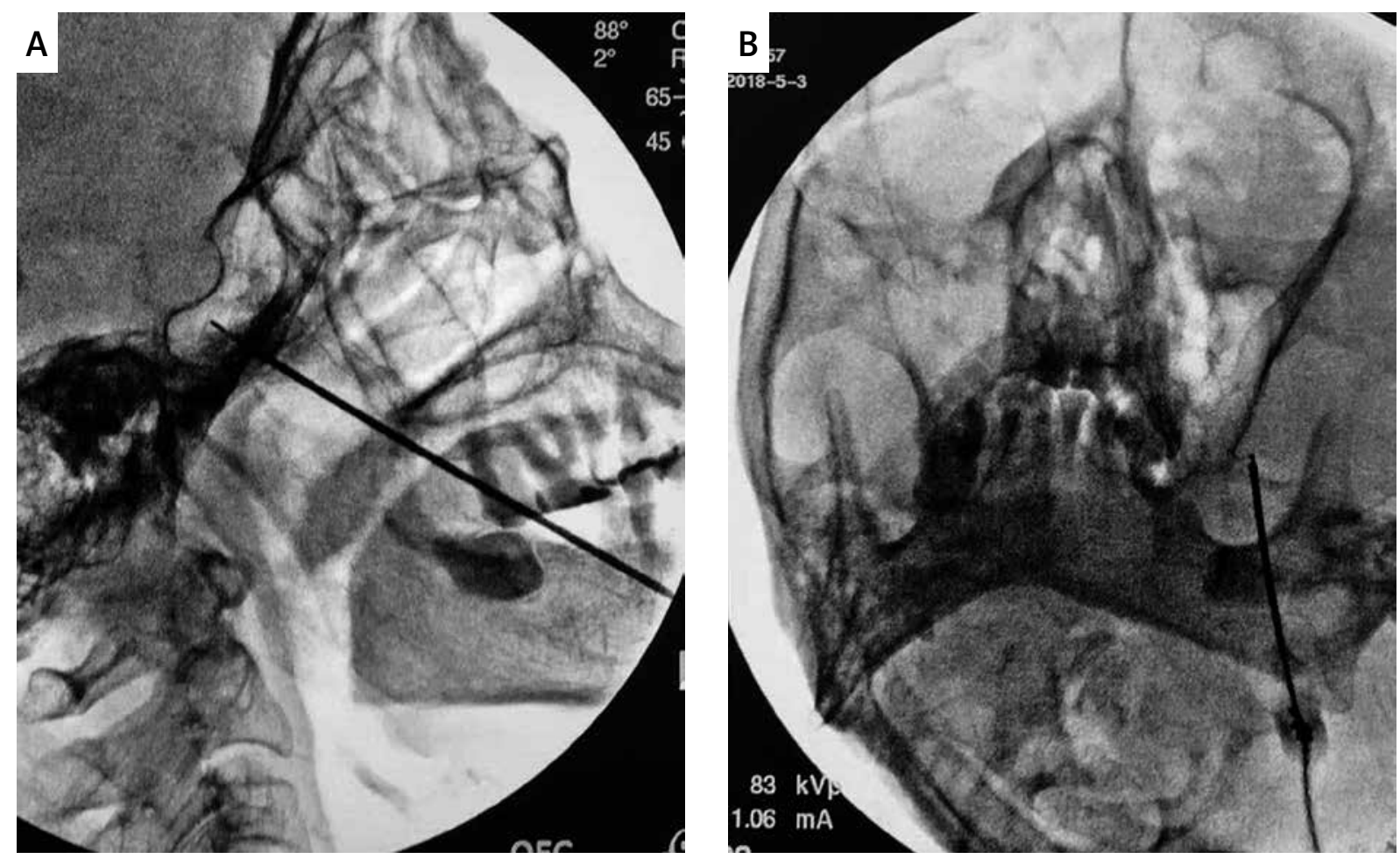

Photo 1. A - Lateral view of C-arm showed that the depth of the needle tip was not beyond the clivus. B - For the coaxial view, the preset coronal and sagittal angles of the X-ray tube to reveal the foramen ovale (FO) were $10^{\circ}$ and $10^{\circ}$ 
after local anesthesia with $0.5 \%$ lidocaine with the "Haertel" approach. During the puncture procedure, advancement was stopped when the needle entered the FO, and the coblation wand (UNITEC, China America United Technology (Beijing) Co. Ltd, Beijing, China) was inserted into the guide needle and extended approximately $5 \mathrm{~mm}$ beyond its tip. The correct coblation wand position was checked radiologically in coaxial and lateral views, and the depth of the tip of the coblation wand was not beyond the clivus. Test coagulation for $0.5-1 \mathrm{~s}$, with the radiofrequency controller set at intensity setting \#1, was performed to re-adjust the coblation wand position with the patient awake and responsive. Intravenous anesthesia with propofol (1-2 mg/kg) and fentanyl (50 $\mu \mathrm{g})$ was administered after verifying the final position of the wand tip, and the ablation of ganglion was performed at intensity setting \#2 for a duration of $30 \mathrm{~s}$. Depending on the nerve branch and the physician's judgment, ablation was performed 2 or 3 times. After

Table I. Numbness rating classification

\begin{tabular}{|lr|}
\hline Grade & Performance \\
\hline 0 & No numbness \\
\hline I & No obvious numbness \\
\hline II & Mild numbness, occasionally affects daily life \\
\hline III & Moderate numbness, frequently affects daily life \\
\hline IV & Painful numbness, severely affect daily life \\
\hline
\end{tabular}

Table II. Characteristics of patients

\begin{tabular}{|c|c|c|}
\hline \multirow[t]{2}{*}{ Parameter } & \multicolumn{2}{|c|}{ Group } \\
\hline & RFT & $\mathrm{CB}$ \\
\hline Patients $(n)$ & 25 & 25 \\
\hline Gender (women/men), $n$ (\%) & $19(76) / 6(24)$ & $14(56) / 11(44)$ \\
\hline Age [years] & $62.56 \pm 11.23$ & $62.88 \pm 13.84$ \\
\hline Presurgery VAS & $7.48 \pm 1.33$ & $7.68 \pm 1.18$ \\
\hline \multicolumn{3}{|l|}{ Branches affected: } \\
\hline V2 & 15 & 8 \\
\hline V3 & 10 & 17 \\
\hline \multicolumn{3}{|l|}{ Pain side: } \\
\hline Right & 17 & 19 \\
\hline Left & 8 & 6 \\
\hline Both & 0 & 0 \\
\hline
\end{tabular}

RFT - radiofrequency thermocoagulation, CB - coblation, V2 - maxillary division, V3 - mandibular division. Data are presented as numbers (\%) of patients or mean $\pm S D$.
PT-RFT or coblation, all patients were asked to remain in bed for $48 \mathrm{~h}$ to prevent intracranial hypotension headache.

Preoperative data, including age, gender, location of pain and preoperative visual analog scale (VAS) score, were recorded. Follow-up evaluation was performed on the first day and at the end of the first month, third month, sixth month and first year after surgery. To assess the degree of numbness, the numbness rating scale described in our previous report was used [8] (Table I).

\section{Statistical analysis}

In this study, a sample size of 25 per group was used to obtain a power of $90 \%$ to show a difference of 6 points on the VAS between the two groups at a significance level of $5 \%$ (two-tailed). Taking into account a potential dropout rate of 10\% (3 patients), we increased the sample size to 28 per group. All data were analyzed with SPSS version 19.0, using mean and standard deviation, with $t$-test and ranksum test for post hoc comparisons. Statistical significance was accepted at $p<0.05$.

\section{Results}

A total of 50 patients with TN were ultimately included in this study (3 cases were dropped from each group for logistical reasons). The basic preoperative data for the two groups are shown in Table II. There were no significant differences in gender, age, pain location or preoperative VAS score between the two groups.

Post-surgery VAS scores were significantly lower in both groups compared with pre-surgery scores $(p<0.05)$, indicating that both methods effectively alleviated pain. At each time point, from 1 day to 1 year, the VAS scores and other parameters were not significantly different between the two groups $(p>0.05)$ (Figure 1).

Among the patients, 19 (76\%) developed different degrees of facial numbness (grades II-IV) after PT-RFT, while 13 (52\%) experienced facial numbness after coblation. One patient in the PT-RFT group experienced painful numbness (grade IV), which subsided to grade III 1 year after surgery, while no patient exhibited painful numbness in the coblation group. The rank-sum test for facial numbness degree showed that the patients in the PT-RFT group experienced more severe numbness than the patients in 
the coblation group, and these differences were statistically significant $(p<0.05)$ (Table III).

Additional post-procedural complications included masseter weakness in $4(9.1 \%)$ patients in the PT-RFT group and in 3 patients in the coblation group. In addition, 1 patient in the PT-RFT group developed hypogeusia, and 1 patient had dysacusis after surgery. All patients improved during follow-up. No mortality was observed in these 50 patients.

\section{Discussion}

PT-RFT is widely used in the treatment of TN when drugs are ineffective or when adverse effects are intolerable [9]. As a minimally invasive technique, PT-RFT has the same efficacy as microvascular decompression (MVD), but with lower surgical risk [10]. However, postoperative facial numbness, a major complication of PT-RFT, limits this procedure [11]. The high temperatures, of over $65^{\circ} \mathrm{C}$, are thought to selectively destroy the $A \delta$ and $C$ pain fibers, as these are more susceptible to heat damage than $A \alpha$ and $A \beta$ tactile fibers [12]. Thus, the facial numbness after PT-RFT might be caused by the heat produced by the PT-RFT needle. In contrast to PT-RFT, coblation technology uses radiofrequency energy to excite the electrolytes in a conductive medium such as saline solution [13]. The coblation needle produces energized plasma that can break molecular bonds, causing tissue dissolution at a relatively low temperature $\left(40-70^{\circ} \mathrm{C}\right)[14,15]$.

Recently, coblation has been used in pain management for different conditions [6, 14-16]. In a case report of phantom limb pain, Li et al. found that coblation of the cervical nerve root successfully reduced the degree of pain [16]. Yang et al. reported on the efficacy of coblation of the thoracic paraver-

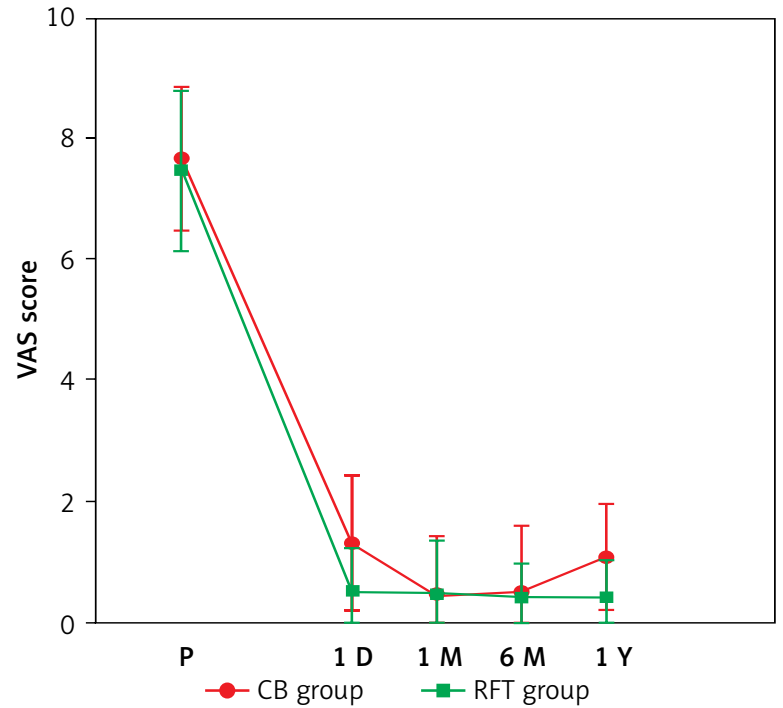

Figure 1. Postprocedure VAS scores between two groups

$C B$ - coblation, RFT - radiofrequency thermocoagulation, $P$ - presurgery.

tebral nerve for the treatment of thoracic neuropathic pain, and found that most patients experienced significant pain relief after surgery. Furthermore, all patients reported slight numbness after surgery, but the degree of numbness had no major impact on their daily lives [6]. Li et al. made a preliminary evaluation of the short-term efficacies of coblation and PT-RFT in treating ITN, and found that coblation reduced the risk of postoperative numbness, while reducing pain to the same degree as PT-RFT [7].

In this report, we compared the efficacy and rates of postoperative facial numbness of PT-RFT and coblation. Compared with patients in the PT-RFT group, the patients in the coblation group experienced similar pain relief, but with milder numbness; this was probably because the lower operating temperatures of coblation are less likely to damage surrounding nerves.

Table III. Comparison of degree of postoperative facial numbness in the 2 groups

\begin{tabular}{|lcccccccc|}
\hline Numbness & \multicolumn{4}{c}{ Group RF } \\
\cline { 2 - 9 } & $1 \mathrm{D}$ & $1 \mathrm{M}$ & $6 \mathrm{M}$ & $1 \mathrm{Y}$ & $1 \mathrm{D}$ & $1 \mathrm{M}$ & $6 \mathrm{M}$ & $1 \mathrm{Y}$ \\
\hline Grade 0 & 0 & 0 & 0 & 0 & 0 & 0 & 0 & 1 \\
\hline Grade I & 6 & 6 & 6 & 6 & 12 & 13 & 19 & 19 \\
\hline Grade II & 9 & 12 & 11 & 13 & 12 & 10 & 6 & 4 \\
\hline Grade III & 9 & 6 & 7 & 6 & 1 & 2 & 0 & 1 \\
\hline Grade IV & 1 & 1 & 1 & 0 & 0 & 0 & 0 & 0 \\
\hline
\end{tabular}


Furthermore, the coblation needle is surrounded by normal saline as a conductive medium, which may also reduce damage to the surrounding tissue.

\section{Conclusions}

Coblation has a similar efficacy in reducing pain as PT-RFT, but with a lower rate of postoperative facial numbness. Therefore, coblation might be a better choice for treating TN.

\section{Conflict of interest}

The authors declare no conflict of interest.

\section{References}

1. Zheng S, Wu B, Zhao Y, et al. Masticatory muscles dysfunction after CT-guided percutaneous trigeminal radiofrequency thermocoagulation for trigeminal neuralgia: a detailed analysis. Pain Pract 2015; 15: 712-9.

2. Maarbjerg S, Di Stefano G, Bendtsen L, et al. Trigeminal neuralgia - diagnosis and treatment. Cephalalgia 2017; 37: 648-57.

3. Maesawa S, Salame C, Flickinger JC, et al. Clinical outcomes after stereotactic radiosurgery for idiopathic trigeminal neuralgia. J Neurosurg 2001; 94: 14-20.

4. Jin HS, Shin JY, Kim YC, et al. Predictive factors associated with success and failure for radiofrequency thermocoagulation in patients with trigeminal neuralgia. Pain Physician 2015; 18: 537-45.

5. Tang YZ, Wu BS, Yang LQ, et al. The long-term effective rate of different branches of idiopathic trigeminal neuralgia after single radiofrequency thermocoagulation: a cohort study. Medicine 2015; 94: e1994.

6. Yang LQ, Gong WY, Wang XP, et al. Computed tomography-guided percutaneously controlled ablation of the thoracic paravertebral nerve due to thoracic neuropathic pain. Pain Pract 2017; 17: 792-9.

7. Li Y, Guo Y, Yang L, et al. Comparison of the short-term outcomes after low-temperature plasma radiofrequency ablation (coblation) in the Gasserian ganglion for the treatment of idiopathic trigeminal neuralgia. J Pain Res 2019; 12: 1235-42.

8. Tang YZ, Jin D, Bian JJ, et al. Long-term outcome of computed tomography-guided percutaneous radiofrequency thermocoagulation for classic trigeminal neuralgia patients older than 70 years. I Craniofac Surg 2014; 25: 1292-5.

9. Kosugi S, Shiotani M, Otsuka Y, et al. Long-term outcomes of percutaneous radiofrequency thermocoagulation of gasserian ganglion for 2nd- and multiple-division trigeminal neuralgia. Pain Pract 2015; 15: 223-8.

10. Tang YZ, Jin D, Li XY, et al. Repeated CT-guided percutaneous radiofrequency thermocoagulation for recurrent trigeminal neuralgia. Eur Neurol 2014; 72: 54-9.

11. Lopez BC, Hamlyn PJ, Zakrzewska JM. Systematic review of ablative neurosurgical techniques for the treatment of trigeminal neuralgia. Neurosurgery 2004; 54: 973-83.
12. Kanpolat Y, Savas A, Bekar A, et al. Percutaneous controlled radiofrequency trigeminal rhizotomy for the treatment of idiopathic trigeminal neuralgia: 25-year experience with 1,600 patients. Neurosurgery 2001; 48: 524-34.

13. Li S, Chen R, Chen Y, et al. Therapeutic effects and safety of percutaneous disc decompression with coblation nucleoplasty in cervical vertigo: a retrospective outcome study with 74 consecutive patients and minimum 1-year follow-up. Pain Physician 2019; 22: E205-14.

14. Azzazi A, AlMekawi S, Zein M. Lumbar disc nucleoplasty using coblation technology: clinical outcome. J Neurointerv Surg 2011; 3: 288-92.

15. Zeng Y, Wang X, Guo Y, et al. Coblation of femoral and sciatic nerve for stump pain and phantom limb pain: a case report. Pain Pract 2016; 16: E35-41.

16. Li H, Li Y, Guo Z, et al. Low-temperature plasma radiofrequency ablation in phantom limb pain: a case report. Brain Circ 2018; 4: $62-4$.

Received: 2.11.2019, accepted: 6.12.2019. 